\title{
Diagnostic Accuracy of Transthoracic Echocardiography and Transesophageal Echocardiography for Ruptured Chordae Tendineae: A Meta-Analysis
}

\author{
Lisi Liao \\ Shenzhen People's Hospital \\ Fa-jin DONG \\ Shenzhen People's Hospital \\ Jie-ying ZENG \\ Shenzhen People's Hospital \\ Bo-bo SHI \\ Shenzhen People's Hospital \\ Xiao-fang ZHONG \\ Shenzhen People's Hospital \\ Yu-xiang HUANG \\ Shenzhen People's Hospital \\ Li-xin CHEN \\ Shenzhen People's Hospital \\ Jinfeng Xu ( $\sim$ 690492148@qq.com) \\ Shenzhen people's hospital
}

\section{Research}

Keywords: Meta-analysis, Echocardiography, Ruptured Chordae Tendineae (RCT), Diagnostic accuracy

Posted Date: May 29th, 2020

DOI: https://doi.org/10.21203/rs.3.rs-29876/v1

License: (c) (i) This work is licensed under a Creative Commons Attribution 4.0 International License. Read Full License 


\section{Abstract \\ Background}

Echocardiography is significant for the diagnosis of ruptured chordae tendineae (RCT) for which transesophageal echocardiography (TEE) is always better than transthoracic echocardiography (TTE), but the diagnostic accuracy of TTE still remains confusing.

\section{Methods}

A meta-analysis of included papers was performed to evaluate the diagnostic accuracy of TTE and TEE, with surgical findings of RCT as the gold standard of patients with suspected RCT.

\section{Results}

The literature search yielded 862 papers, 6 met the inclusion criteria, included 505 patients, for detecting RCT, the sensitivity and specificity of TTE were $48 \%$ (95\% Cl: $35-62 \%)$ and $98 \%$ (95\% Cl: 89-100\%), and those of TEE were 99\% (95\% Cl: $64-100 \%)$ and $94 \%$ (95\% Cl: $87-98 \%)$, respectively. The summary + LR, -LR of TTE were 22.40 (95\% Cl: $4.89-102.62), 0.53(95 \% \mathrm{Cl}: 0.41-0.68)$, respectively. The summary + LR, -LR of TEE were 17.62(95\%Cl: 7.16-43.39), 0.02(95\%Cl:0. 00-0.57), respectively. For TTE, the area under SROC was $84 \%(95 \% \mathrm{Cl}: 92 \%-96 \%)$,and that of TEE is $98 \%$. The pooled + LR and -LR were calculated by setting the prior probabilities of $20 \%$ in both TTE and TEE.

\section{Conclusions}

TTE is not highly sensitive for the detection of RCT but is highly specific, so there is a potential of missing diagnosis, and subsequent TEE is almost required in highly suspected patients.

\section{Background}

Ruptured Chordae Tendineae (RCT) is increasingly reported as one of the important causes of severe mitral regurgitation (MR)[1], which is a progressive disease with acute clinical symptoms that eventually requires mitral valve (MV) surgery[2].

Echocardiography is widely used in diagnosing RCT, which has an irreplaceable position[3]. The definite diagnosis plays a decisive role in the formulation of treatment plans. It is generally known that the methods we used to detect RCT include transesophageal echocardiography (TEE) and transthoracic echocardiography (TTE). However, compared with TTE, TEE has better views and intensive resources, but uncomfortable for patients and associated with complication risks [4]. Under this situation, TTE is particularly important for the diagnosis of RCT.

A series of studies shown the diagnostic performance of TTE and TEE for RCT. Previous studies demonstrated that the sensitivity of TEE was higher than that of TTE for the detection of RCT, which could reach $100 \%[5,6]$. Some of the studies showed the high sensitivity of TTE for the diagnosis of RCT, the highest could reach $90 \%$ [7]. However, others showed the low sensitivity of TTE for detection of RCT, the lowest was $0 \%$ [6]. According to the large range differences in sensitivity of TTE, we want to evaluate the actual value of TTE and TEE in the identification of the accuracy of the RCT causing MR.

Therefore, we conducted this meta-analysis to evaluate the diagnostic value of TTE and TEE using RCT findings on surgical as the gold standard. And discussing whether TEE can be replaced by TTE in diagnosing RCT.

\section{Methods}

\section{Search strategy}

We searched the databases of Embase, Pubmed and Cochrane library for relevant English studies up to July 20, 2019. Terms of "Chordae Tendinae" or "Chorda Tendinea rupture", "echocardiography", "sensitivity" OR "specificity specificity" OR 'diagnostic accuracy' OR 'surgery' were used. Moreover, the reference lists of the retrieved systematic and narrative reviews were also manually searched to identify additional relevant studies.

\section{Study selection}


Studies were included if they met all of the following criteria: 1 . clinically suspected MR due to any cause, 2 . surgery findings as the gold standard for RCT, 3. sufficient data to allow extraction of a $2 \times 2$ diagnostic table, 4. papers after the year of 1989. Reviews, letters, case reports and commentaries were excluded.

Two authors (Dong, FJ and Liao, LS) with the same expertise and experience selected the eligible studies independently. Discrepancies between them were resolved by the 3rd author (Chen LX).

\section{Data Extraction and Quality evaluation}

All papers were screened by titles and abstracts. Full text and reference lists of relevant papers fulfilling the inclusion and exclusion criteria were reviewed in detail. Data for $2 \times 2$ tables were extracted.

For each included full-text article, quality was evaluated by the Quality Assessment of Diagnostic Accuracy Studies-2 (QUADAS-2) or potential biases.

\section{Statistical analysis}

For detections of RCT on TTE and TEE, specificity, sensitivity, and likelihood ratios were calculated from the abstracted data for each study.

All the analysis was performed by Stata 14.0 (Stata Corp, College Station, TX, USA) with Midas module was used to pool statistics indexes and draw statistical graphs for TTE and TEE separately, such as sensitivity, specificity, the pooled forest graph of the negative likelihood ratio (NLR), the positive likelihood ratio (PLR), and the diagnostic odds ratio(DOR) with corresponding $95 \% \mathrm{Cl}$ (confidence intervals), the area under the summary receiver operating characteristic curve (SROC). $95 \% \mathrm{Cl}$ was calculated for specificity, sensitivity and likelihood ratios using standard methods. SROC was used to examine the diagnostic value of TTE and TEE. Threshold effect was tested by SROC and Spearman correlation coefficient.

RevMan 5.3 (The Nordic Cochrane Center, The Cochrane Collaboration, Copenhagen, Denmark) was used to evaluate the included papers. The inconsistency index $\left(\mathrm{I}^{2}\right)$ and Cochrane $\mathrm{Q}$ statistic $(\mathrm{p})$ were used to estimate the heterogeneity across the included studies. If Cochran $Q$ statistic value $p>0.1$ or inconsistency index $I^{2}<50 \%$, a fixed-effect model will be used; otherwise, a random effects model was selected. Deek's funnel plot asymmetry test will be used to test the potential publication bias, with $p<0.10$ for the slope coefficient indicating significant asymmetry.

\section{Results}

\section{Literature searches}

We yielded 862 papers, after excluded the duplications, then screened by title and abstract, finally 15 papers were assessed by full-text for eligibility. 5 papers were further excluded, due to analyzed the TTE or TEE only. 3 papers were further excluded because the authors compared TTE with 3DTEE. 1 articles did not contain enough information to extract $2 \times 2$ table for analysis. Therefore, 6 studies were ultimately met the inclusion criteria. The screening flow was shown in Fig. 1. 
Table 1

Characteristics of the included studies

\begin{tabular}{|c|c|c|c|c|c|c|c|c|c|c|c|c|c|c|}
\hline \multirow[t]{2}{*}{ Study } & \multirow[t]{2}{*}{ Year } & \multirow[t]{2}{*}{$\mathrm{M} / \mathrm{F}$} & \multirow[t]{2}{*}{ Age } & \multirow[t]{2}{*}{ Country } & \multirow{2}{*}{$\begin{array}{l}\text { Type of } \\
\text { study }\end{array}$} & \multirow[t]{2}{*}{ Patients(RCT) } & \multicolumn{4}{|c|}{ TTE } & \multicolumn{4}{|c|}{ TEE } \\
\hline & & & & & & & $\mathrm{TP}$ & $\mathrm{FP}$ & $\mathrm{FN}$ & $\mathrm{TN}$ & TP & FP & $\mathrm{FN}$ & $\mathrm{TN}$ \\
\hline $\begin{array}{l}\text { Hozumi, et } \\
\text { al. }\end{array}$ & 1990 & $12 / 16$ & $\begin{array}{l}27- \\
77\end{array}$ & Japan & Prospective & $28(17)$ & 6 & 0 & 11 & 11 & 17 & 0 & 0 & 11 \\
\hline Alam,et al. & 1991 & - & - & America & Prospective & $23(6)$ & 0 & 0 & 6 & 17 & 6 & 0 & 0 & 17 \\
\hline $\begin{array}{l}\text { Sochowski, } \\
\text { et al. }\end{array}$ & 1991 & $20 / 7$ & - & Canada & Retrospective & $27(20)$ & 12 & 0 & 8 & 7 & 20 & 1 & 0 & 6 \\
\hline Shyu, et al. & 1992 & $43 / 29$ & $\begin{array}{l}21- \\
75\end{array}$ & Taiwan & Prospective & 72(52) & 26 & 2 & 14 & 18 & 40 & 2 & 0 & 18 \\
\hline $\begin{array}{l}\text { Hellemans, } \\
\text { et al. }\end{array}$ & 1996 & - & $\begin{array}{l}16- \\
82\end{array}$ & Netherlands & Prospective & 294(86) & 49 & 8 & 37 & 200 & 68 & 8 & 18 & 200 \\
\hline $\begin{array}{l}\text { Minami, et } \\
\text { al. }\end{array}$ & 2012 & $30 / 31$ & $\begin{array}{l}22- \\
77\end{array}$ & Japan & Prospective & 61(39) & 17 & 0 & 22 & 22 & 29 & 4 & 10 & 18 \\
\hline \multicolumn{15}{|c|}{ M/F: male/ female; RCT: ruptured chordae tendineae; } \\
\hline \multicolumn{15}{|c|}{ TTE : transthoracic echocardiography; TEE: transesophageal echocardiography; } \\
\hline TP : true-p & TS & $n$ & . & lconncit & l. falco & & & & & & & & & \\
\hline
\end{tabular}

\section{Study characteristics}

The 6 studies included 505 patients with suspected RCT, of whom all had MV on both TTE and TEE. Study size ranged from 23 patients to 294 patients. The sensitivity of patients with RCT who underwent TTE ranged from $0-65 \%$, that of TEE ranged from $74-100 \%$.

\section{Methodology quality assessment}

The QUADAS-2 checklist was used. The included studies' quality was judged to be high (Fig. 2). All the quality assessment items of the included studies had a low risk of bias.

\section{Data synthesis and analysis}

A random effects model was used. Significant heterogeneity of TTE in pooling the sensitivity $\left(I^{2}=63.07 \%, p=0.02\right)$ and that of TEE $\left(I^{2}=\right.$ $90.01 \%, p=0.00)($ Fig. 3).

The pooled sensitivity and specificity of TTE were $48 \%$ (95\% Cl: 35-62\%) (Fig. 3A) and 98\% (95\% Cl: 89-100\%) (Fig. 3B), and those of TEE were 99\% (95\%Cl: 64-100\%) (Fig. 3A) and 94\%(95\% Cl: 87-98\%) (Fig. 3B), respectively. The pooled + LR, -LR of TTE were 22.40 (95\%Cl 4.89102.62), 0.53 (95\% Cl: $0.41-0.68)$ respectively (Fig. $4 \mathrm{~A})$. As that of TEE were 17.62 (95\%Cl: $7.16-43.39), 0.02(95 \% \mathrm{Cl}: 0.00-0.57)$, respectively(Fig. 4B). For TTE, the area under SROC was $84 \%(95 \% \mathrm{Cl}$ : $92 \%-96 \%)$ (Fig. 5),and that of in TEE is $98 \%$. The pooled -LR and + LR were calculated by setting $20 \%$ prior probabilities in both TTE and TEE (Fig. 6).

\section{Publication bias}

Publication bias was tested by Deeks' funnel plot, showed that studies were distributed symmetrically and no clear evidence of publication bias ( $p=0.10$ in TTE, and 0.43 in TEE, Fig. 7). Regression test of InDOR against 1/Effective Sample Size 1/2 indicated no obvious small-study bias in our meta-analysis.

\section{Discussion}

Our studies findings demonstrated that for detecting RCT in all patients, TTE had a sensitivity of $48 \%$ and specificity of $98 \%$, so it has a likelihood to miss almost $50 \%$ potential RCTs. For TEE, the sensitivity and specificity of it can reach a high level, which is $99 \%$ and $94 \%$ respectively. That means TEE is pretty matched with surgical findings of previous studies [8]. Monin.et al. [7]reported the diagnosis accuracy of RCT confirmed by TTE could reach 90\%. Conversely, Alam.et al. [6] reported that RCT was seen in none of 23 patients (sensitivity = $0 \%$ ). In 
brief, the reported sensitivity of TTE for detecting RCT has varied considerably. Herein, our study of 6 observational studies, which showed differences in sensitivity of TTE for the diagnosis of RCT that ranged from $0-65 \%[3,5,6,9-11]$. The significant heterogeneity based on I2 for TTE analyses from the Alam in 1991, probably because the sample size is small.

Rupture of mitral chordae tendineae is one of the causes of acute mitral valve regurgitation, which can lead to acute pulmonary edema and cardiogenic shock [12]. It is one of the most common valvular heart diseases, which is secondary to Marfan syndrome, connective tissue disease, coronary heart disease, congenital heart disease, infective endocarditis, rheumatic heart disease and degenerative valvular disease [13]. It often occurs because of the weakness of mitral chordae tendineae, papillary muscles, and abnormal valve position or shape. Primary person refers to the mitral valve leaflet and annular disease caused by degeneration. It is also one of the valvular diseases treated surgically [14]. Early diagnosis of RCT has great significance for treatment, which can save the patient's life. Long-term follow-up shows that patients with RCT and MR are more likely to use valve repair [15]. Valve repair has more advantages than valve replacement, such as lower mortality, higher long-term survival rate, lower risk of thrombus embolism and bleeding, better left ventricular systolic function after the operation. The etiology, lesion location and characteristics of mitral valve prolapse are important factors determining the surgical method, among which, mitral valve posterior lobe prolapse and chordae tendineae ruptures are more suitable for the application of valve repair [16]. Therefore echocardiography it is very significant, in addition to diagnosis, it also needs to understand the location of the diseased lobe and the pathological characteristics of its accessory structure before the operation.

TEE and TTE are important for the diagnosis of RCT [17]. TEE has a better view because the esophagus is closer to the heart, TEE can avoid the interference of thoracic morphology and lung, and the TEE probe has a higher discrimination rate, so it is easier to view the morphology, texture and motor characteristics of the diseased lobe and chordae tendineae [18]. Therefore, the accuracy of TEE in the diagnosis of valvular heart disease is higher than that of TTE [19]. Besides, TEE can have side effects that make patients feel unpleasant as mentioned before, so inspectors should not overuse TEE method to increase the burden on patients. Yu HT et al. [20] did a study in 2013 showed a high prevalence of unrecognized RCT in mitral valve prolapse patients undergoing valve surgery. RCTs were not found in 124 patients, 73 have no RCT observed at surgery, but 51 surgically proved RCT, which means TTE still has a large chance to miss many subtle RCTs even with rapidly developing imaging facilities.

As we have known the typical echocardiography manifestations of mitral valve prolapse and chordae tendineae rupture are the tip of the diseased valve lobe or the chordae tendineae partially ruptured moving back and forth between the atrioventricular in the following cycles [21].The ruptured end of the systolic chordae tendineae was under the lobe of the healthy side and was impacted by the regurgitation tract. Its residual end pointed to the atrial wall or atrial septum of the healthy side, while it returned to the ventricular chamber during diastole [22]. And, when the course of disease gets longer, the calcification of disease change place is heavier; TTE is not easy to detect RCT. Under this situation, TTE it is easier to miss diagnoses.

Combined with our research, we had a finding. Although with the developments in the resolution of TTE over the past 30 years, the sensitivity of TTE to detect RCT has no significant improvement. Complex co-combination of valve conditions occurs with calcification or infectious endocarditis. Maybe TTE has a high sensitivity for the typical signs but not for complicated or untypical signs.

Our study also has several clinical implications. For patients with mitral regurgitation, TTE should be the recommended examination for all patients because of convenient and its high specificity. In these patients, if TTE is highly indicative of RCT, the diagnostic accuracy is high because TTE is highly specific to RCT [23]. Conversely; the absence of valve abnormalities in TTE mostly reduced the likelihood of RCT, so there is no need for TEE. This can eliminate needless hurts that are associated with cost and risk for complication. However, the decision on whether to continue with TEE or not should be determined by the patient's clinical symptoms and doctor. When the valve condition is complex, TTE is difficult to distinguish from the abnormal valve as if due to degenerative mitral valve diseases, vegetation or RCT, TEE should be performed.

This study had several limitations due to potential biases. We included patients that constructed both TTE and TEE could miss many studies that underwent TTE or TEE only.

\section{Conclusions}

TTE is not highly sensitive for the detection of RCT, but is highly specific,so there is a potential of missing diagnosis, and subsequent TEE is almost required in highly suspected patients.

\section{Abbreviations}

RCT: Ruptured Chordae Tendineae; TEE:transesophageal echocardiography; TTE:transthoracic echocardiography; MR:mitral regurgitation; MV:mitral valve 


\section{Declarations}

\section{Availability of data and materials}

The datasets used and/or analyzed during the current study are available from the corresponding author on reasonable request.

\section{Ethics approval and consent to participate}

Not applicable.

\section{Consent for publication}

Not applicable.

\section{Competing interests}

The authors declare that they have no competing interests.

\section{Funding}

Not applicable.

\section{Authors' contributions}

Li-si LIAO and Fa-jin DONG were primary responsible for the conception and design of review. Both authors read and approved the final manuscript.

\section{Acknowledgements}

Not applicable.

\section{References}

1. Carpentier A, Deloche A, Dauptain J, Soyer R, Blondeau P, Piwnica A, Dubost C, McGoon DC. A new reconstructive operation for correction of mitral and tricuspid insufficiency. J Thorac Cardiovasc Surg. 1971;61(1):1-13.

2. DeAnda A Jr, Kasirajan V, Higgins RSD. Mitral valve replacement versus repair in 2003: where do we stand? Curr Opin Cardiol. 2003;18(2):102-5.

3. Minami T, Kawano H, Yamachika S, Tsuneto A, Kaneko M, Kawano Y, Minami S, Eishi K, Maemura K. Comparison of the diagnostic power of transthoracic and transesophageal echocardiography to detect ruptured chordae tendineae. Int Heart J. 2012;53(4):225-9.

4. Hilberath JN, Oakes DA, Shernan SK, Bulwer BE, D'Ambra MN, Eltzschig HK. Safety of transesophageal echocardiography. Journal of the American Society of Echocardiography: official publication of the American Society of Echocardiography. 2010;23(11):1115-27. quiz $1220-1111$.

5. Hozumi T, Yoshikawa J, Yoshida K, Yamaura Y, Akasaka T, Shakudo M. Direct visualization of ruptured chordae tendineae by transesophageal two-dimensional echocardiography. J Am Coll Cardiol. 1990;16(5):1315-9.

6. Alam M, Sun I. Superiority of transesophageal echocardiography in detecting ruptured mitral chordae tendineae. American heart journal. 1991;121(6 Pt 1):1819-21.

7. Monin JL, Dehant P, Roiron C, Monchi M, Tabet JY, Clerc P, Fernandez G, Houel R, Garot J, Chauvel C, et al. Functional assessment of mitral regurgitation by transthoracic echocardiography using standardized imaging planes diagnostic accuracy and outcome implications. J Am Coll Cardiol. 2005;46(2):302-9.

8. Wu W, Luo X, Wang L, Sun X, Jiang Y, Huo S, Tu D, Bai Z, Wang H. The accuracy of echocardiography versus surgical and pathological classification of patients with ruptured mitral chordae tendineae: a large study in a Chinese cardiovascular center. J Cardiothorac Surg. 
2011;6:94.

9. Sochowski RA, Chan KL, Ascah KJ, Bedard P: Comparison of accuracy of transesophageal versus transthoracic echocardiography for the detection of mitral valve prolapse with ruptured chordae tendineae (flail mitral leaflet). The American journal of cardiology 1991 , 67(15):1251-1255.

10. Shyu KG, Lei MH, Hwang JJ, Lin SC, Kuan P, Lien WP. Morphologic characterization and quantitative assessment of mitral regurgitation with ruptured chordae tendineae by transesophageal echocardiography. The American journal of cardiology. 1992;70(13):1152-6.

11. Hellemans IM, Pieper EG, Ravelli AC, Hamer JP, Jaarsma W, van den Brink RB, Peels CH, van Swieten HA, Tijssen JG, Visser CA. Comparison of transthoracic and transesophageal echocardiography with surgical findings in mitral regurgitation. The ESMIR Research Group. The American journal of cardiology. 1996;77(9):728-33.

12. Dolmatova EV, Moazzami K, Maher J, Klapholz M, Sambol J, Waller AH. Chordae Tendineae Rupture in the United States: Trends of Outcomes, Costs and Surgical Interventions. Heart Surg Forum. 2017;20(1):E019-25.

13. Yoshida S, Fukushima S, Miyagawa S, Yoshikawa Y, Hata H, Saito S, Saito T, Domae K, Kashiyama N, Matsuura R, et al. The Adaptive Remodeling of the Anterior Mitral Leaflet and Chordae Tendineae Is Associated with Mitral Valve Function in Advanced Ischemic and Nonischemic Dilated Cardiomyopathy. Int Heart J. 2018;59(5):959-67.

14. Manda J, Kesanolla SK, Hsuing MC, Nanda NC, Abo-Salem E, Dutta R, Laney CA, Wei J, Chang CY, Tsai SK, et al. Comparison of real time two-dimensional with live/real time three-dimensional transesophageal echocardiography in the evaluation of mitral valve prolapse and chordae rupture. Echocardiography. 2008;25(10):1131-7.

15. Hien MD, Grossgasteiger M, Rauch H, Weymann A, Bekeredjian R, Rosendal C. Experts and beginners benefit from three-dimensional echocardiography: a multicenter study on the assessment of mitral valve prolapse. Journal of the American Society of Echocardiography: official publication of the American Society of Echocardiography. 2013;26(8):828-34.

16. Obase K, Weinert L, Hollatz A, Farooqui F, Roberts JD, Minhaj MM, Tung A, Chaney M, Ota T, Jeevanandam V, et al. Elongation of chordae tendineae as an adaptive process to reduce mitral regurgitation in functional mitral regurgitation. Eur Heart $\mathrm{J}$ Cardiovasc Imaging. 2016;17(5):500-9.

17. Dal-Bianco JP, Bartko PE, Levine RA. The power of ultrasound: treating secondary MR with sound waves. Eur Heart $\mathrm{J}$ Cardiovasc Imaging. 2016;17(10):1108-9.

18. Hahn RT, Abraham T, Adams MS, Bruce CJ, Glas KE, Lang RM, Reeves ST, Shanewise JS, Siu SC, Stewart W, et al.

19. Hahn RT, Abraham T, Adams MS, Bruce CJ, Glas KE, Lang RM, Reeves ST, Shanewise JS, Siu SC, Stewart W, et al. Guidelines for performing a comprehensive transesophageal echocardiographic examination: recommendations from the American Society of Echocardiography and the Society of Cardiovascular Anesthesiologists. Journal of the American Society of Echocardiography: official publication of the American Society of Echocardiography. 2013;26(9):921-64.

20. Yu HT, Moon J, Yang WI, Shim CY, Lee S, Chang BC, Hong GR, Ha JW. High prevalence of unrecognized chordae tendineae rupture in mitral valve prolapse patients undergoing valve replacement surgery. Can J Cardiol. 2013;29(12):1643-8.

21. Himelman RB, Kusumoto F, Oken K, Lee E, Cahalan MK, Shah PM, Schiller NB. The flail mitral valve: echocardiographic findings by precordial and transesophageal imaging and Doppler color flow mapping. J Am Coll Cardiol. 1991;17(1):272-9.

22. Takamoto T, Nitta M, Tsujibayashi T, Taniguchi K, Marumo F. [The prevalence and clinical features of pathologically abnormal mitral valve leaflets (myxomatous mitral valve) in the mitral valve prolapse syndrome: an echocardiographic and pathological comparative study]. Journal of cardiology Supplement. 1991;25:75-86.

23. Hien MD, Rauch H, Lichtenberg A, De Simone R, Weimer M, Ponta OA, Rosendal C. Real-time three-dimensional transesophageal echocardiography: improvements in intraoperative mitral valve imaging. Anesthesia analgesia. 2013;116(2):287-95.

\section{Figures}




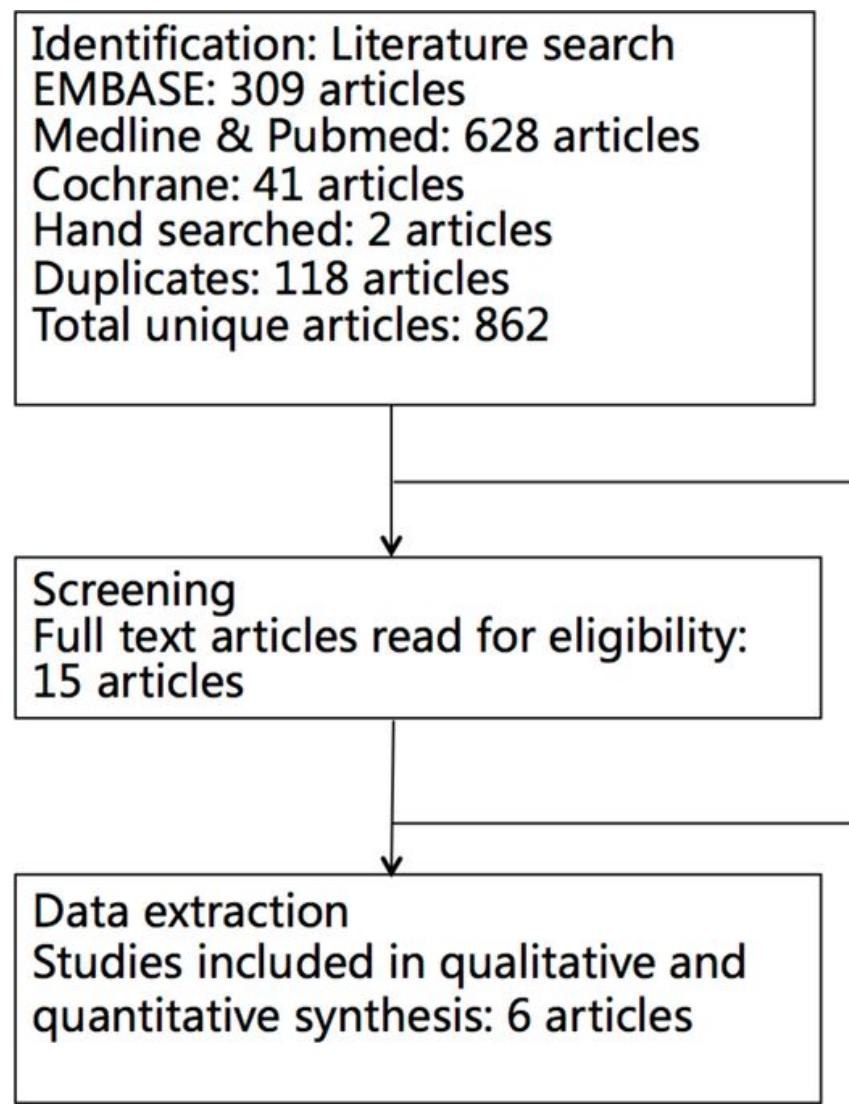

Excluded on screening: 847 articles 230 articles were case reports, commentaries, letters or reviews 18 articles did not involve human subjects

12 articles included only children

24 articles did not use TTE or TEE 525 articles did not compare TTE to TEE 38 articles included only Ruptured Chordae Tendineae cases

Excluded after reading full text: 9 articles 5 analyzed the TTE or TEE only.

1 articles did not contain enough information to extract $2 \times 2$ table for analysis

3 articles compared TTE to 3DTEE

Figure 1

Flow chart of study selection process 


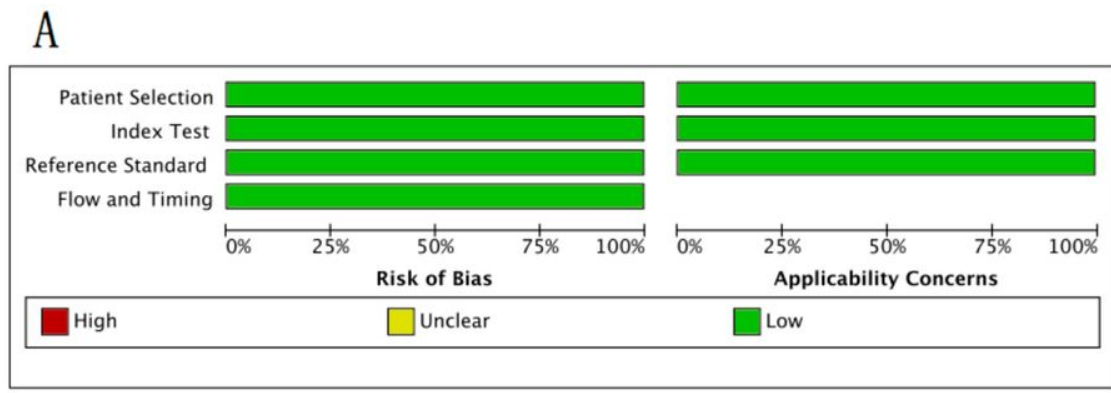

B

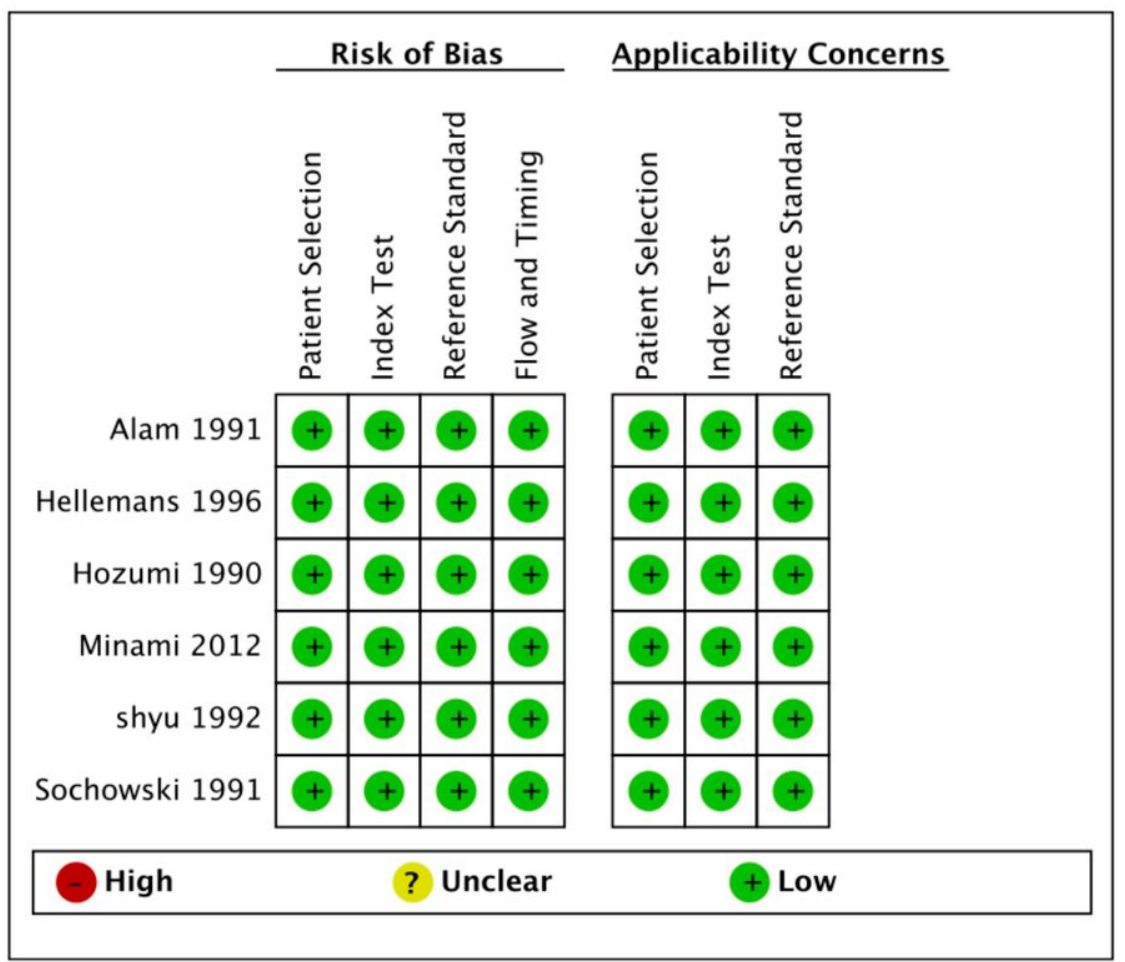

\section{Figure 2}

Quality assessment, by using the Quality Assessment of Diagnostic Accuracy Studies-2 tool. A) Risk of bias and applicability concerns graph: review authors' judgments about each domain presented as percentages across included studies. B) Risk of bias and applicability concerns summary: review authors' judgments about each domain for each included study. 


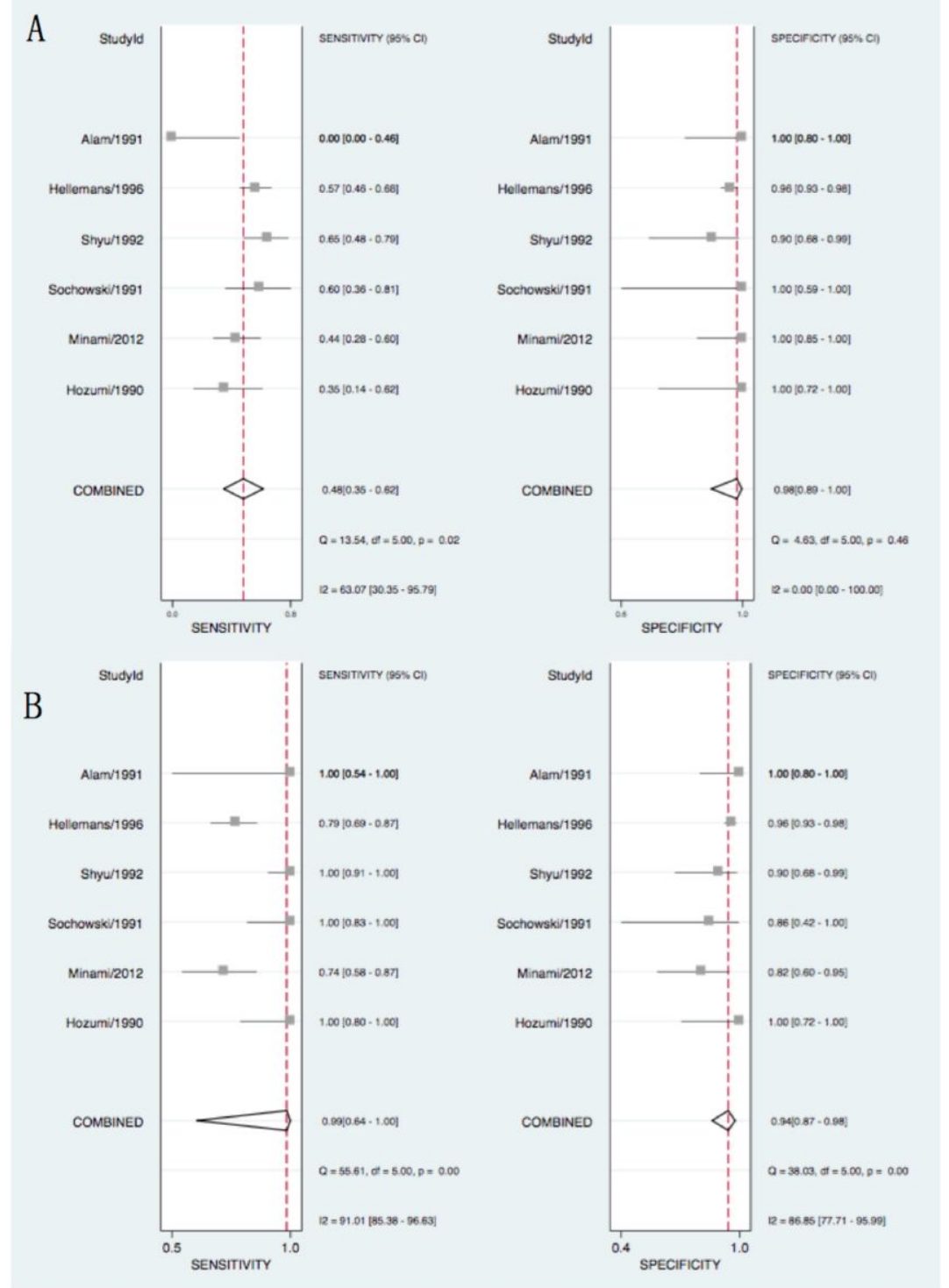

Figure 3

Forest plots for sensitivity and specificity for $\operatorname{TTE}(\mathrm{A})$ and $\operatorname{TEE}(\mathrm{B})$ of diagnosis accuracy of RCT. 


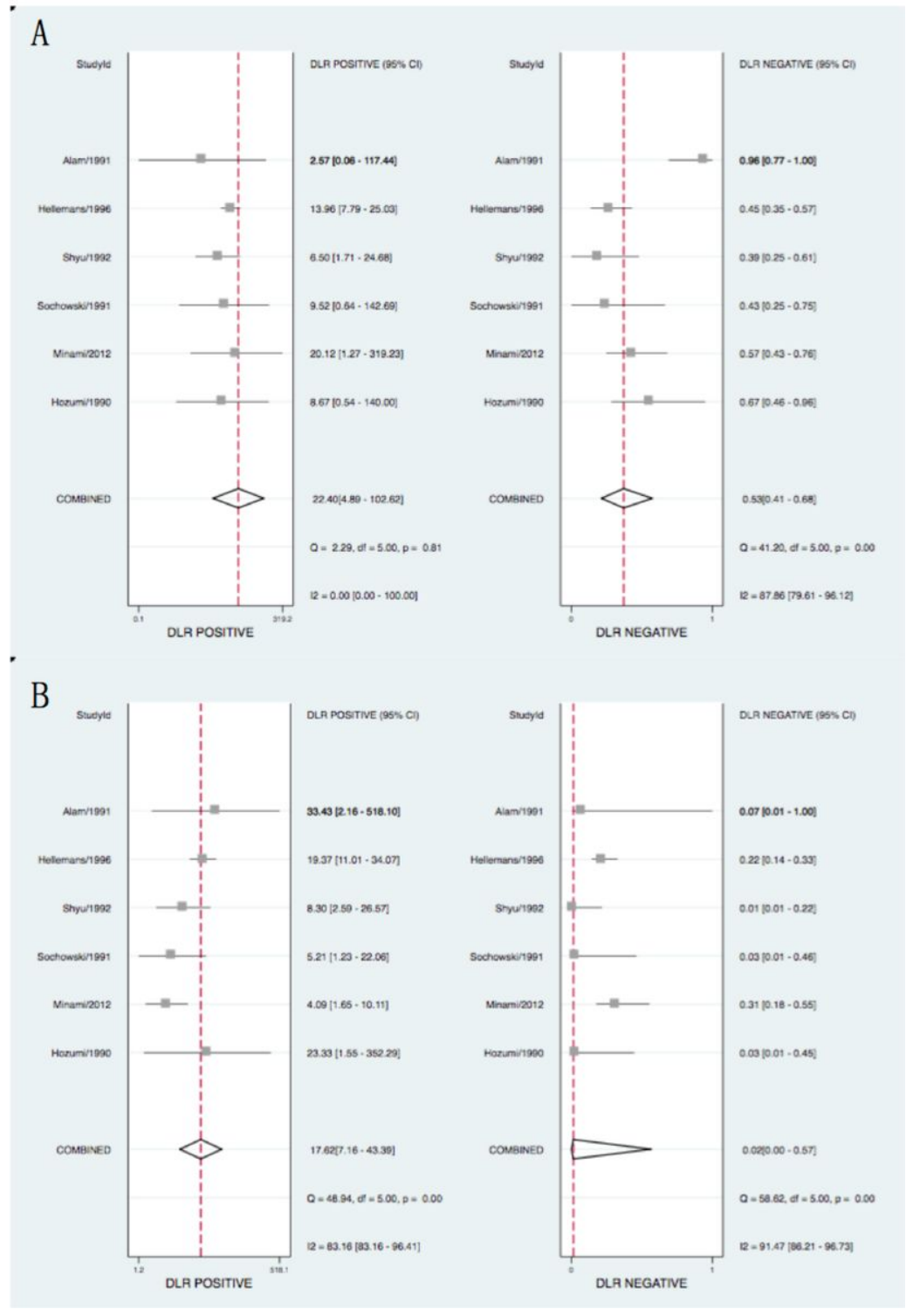

Figure 4

Forest plots for positive likelihood ratio and negative likelihood ratio for TTE(A) and TEE (B). 


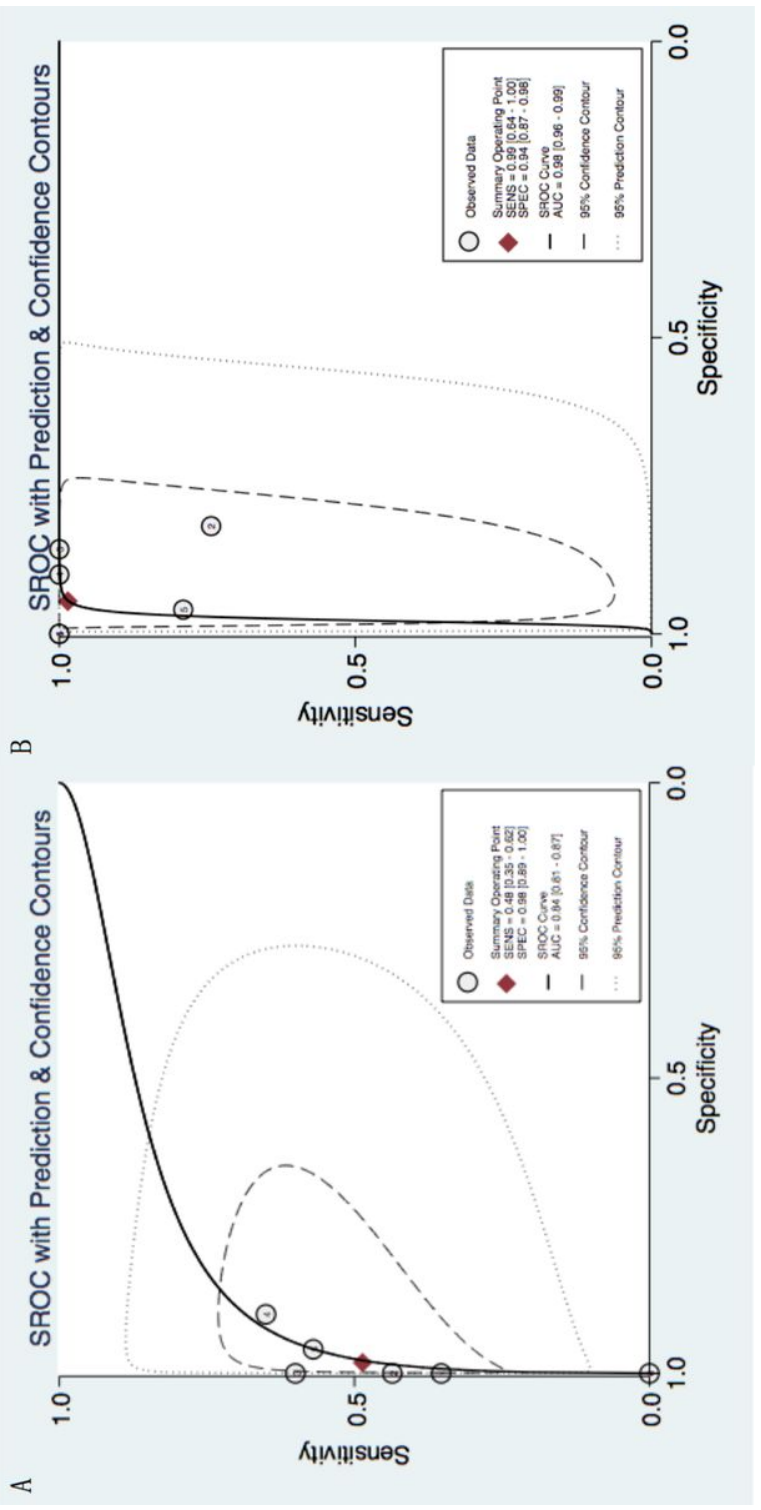

Figure 5

Hierarchical summary receiver operating characteristic curve (HSROC) of diagnosis accuracy in TTE (A) and TEE (B). 
(\%) Kin!lqeqoud ısel-tsod

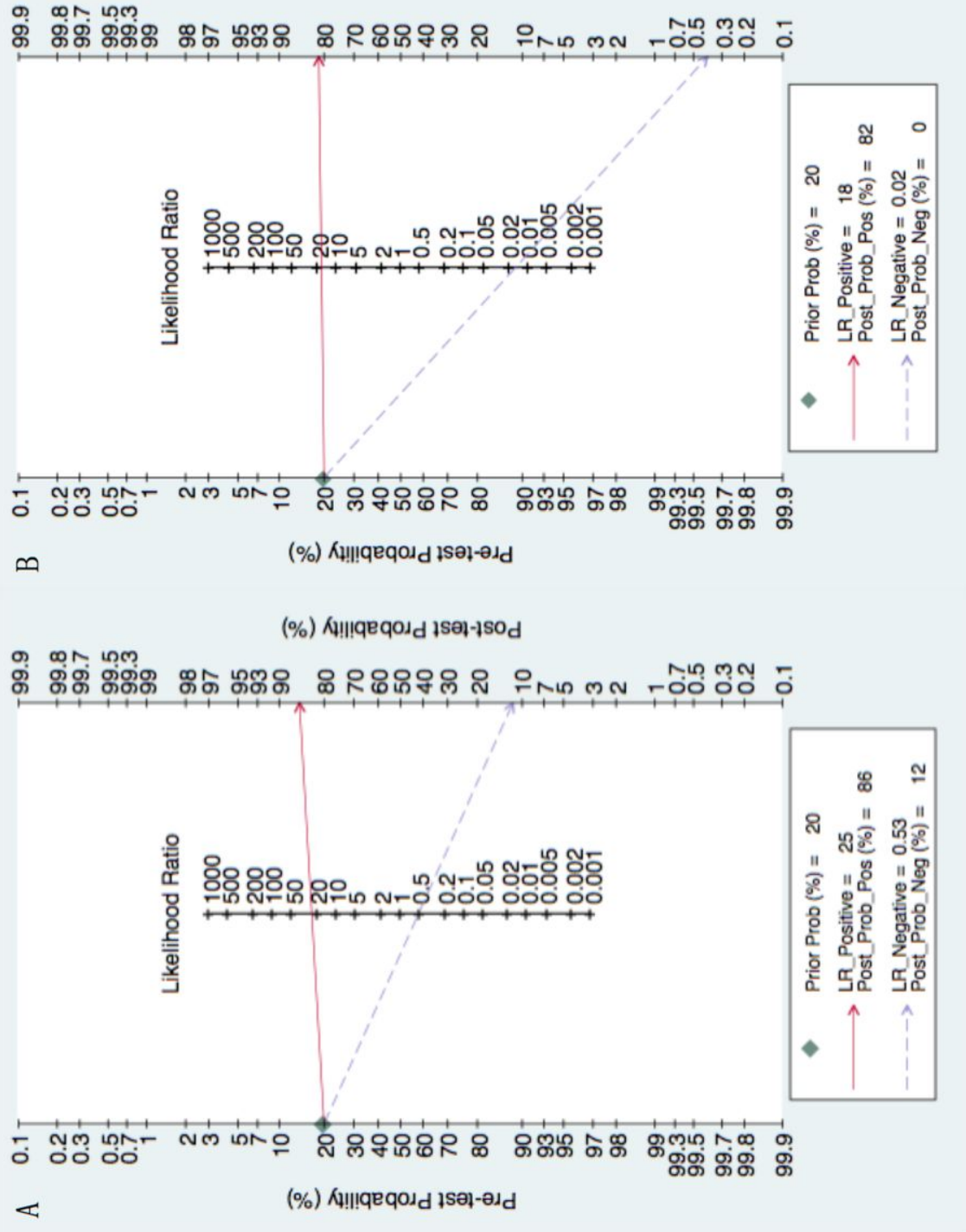

\section{Figure 6}

Fagan's Nomogram shows that virtual touch tissue quantification as a screening tool for diagnostic accuracy in TTE $₫ \mathrm{~A} \rrbracket$ pretest probability at $20 \%$, and TEE囚B囚pretest probability at $20 \%$. 


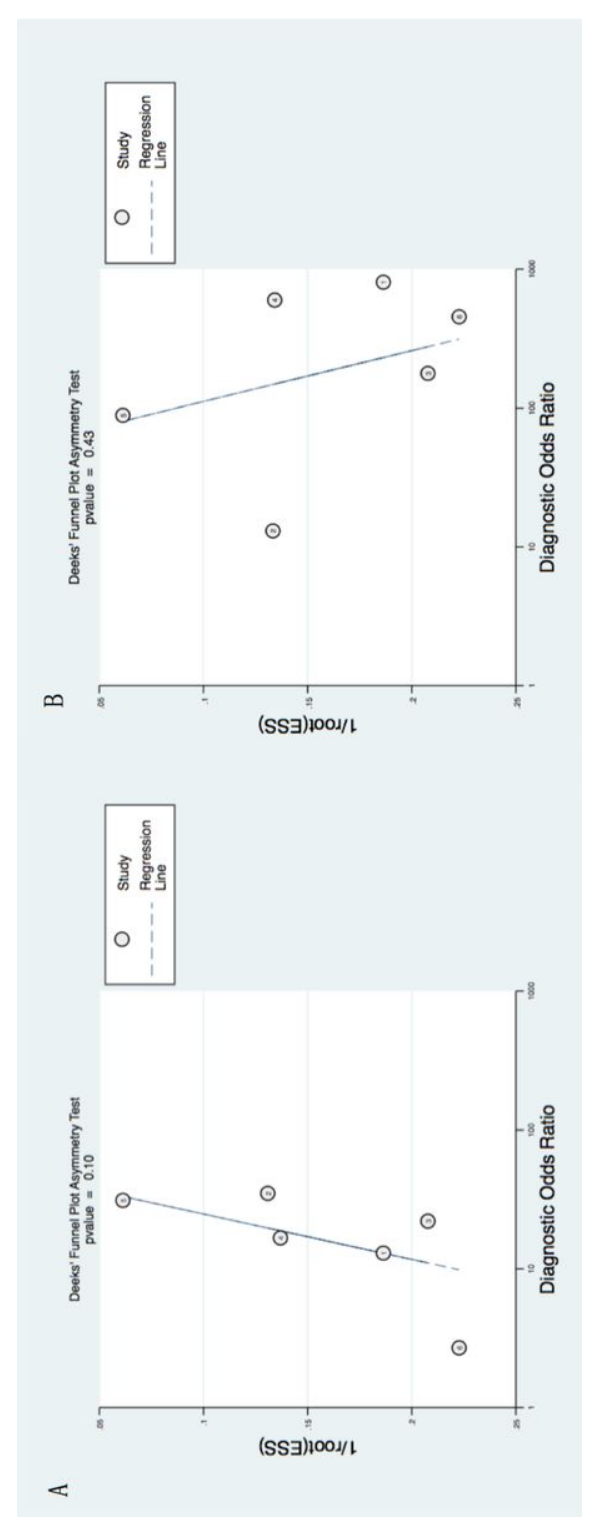

\section{Figure 7}

Deeks' funnel plot with superimposed regression line for identifying publication bias Log Odds Ratio versus 1 sqrt (Effective Sample Size) (Deeks) indicated that no significant bias was found. ESS=effective sample size. 